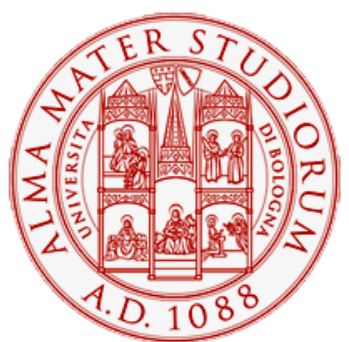

Alma Mater Studiorum - Università di Bologna DEPARTMENT OF ECONOMICS

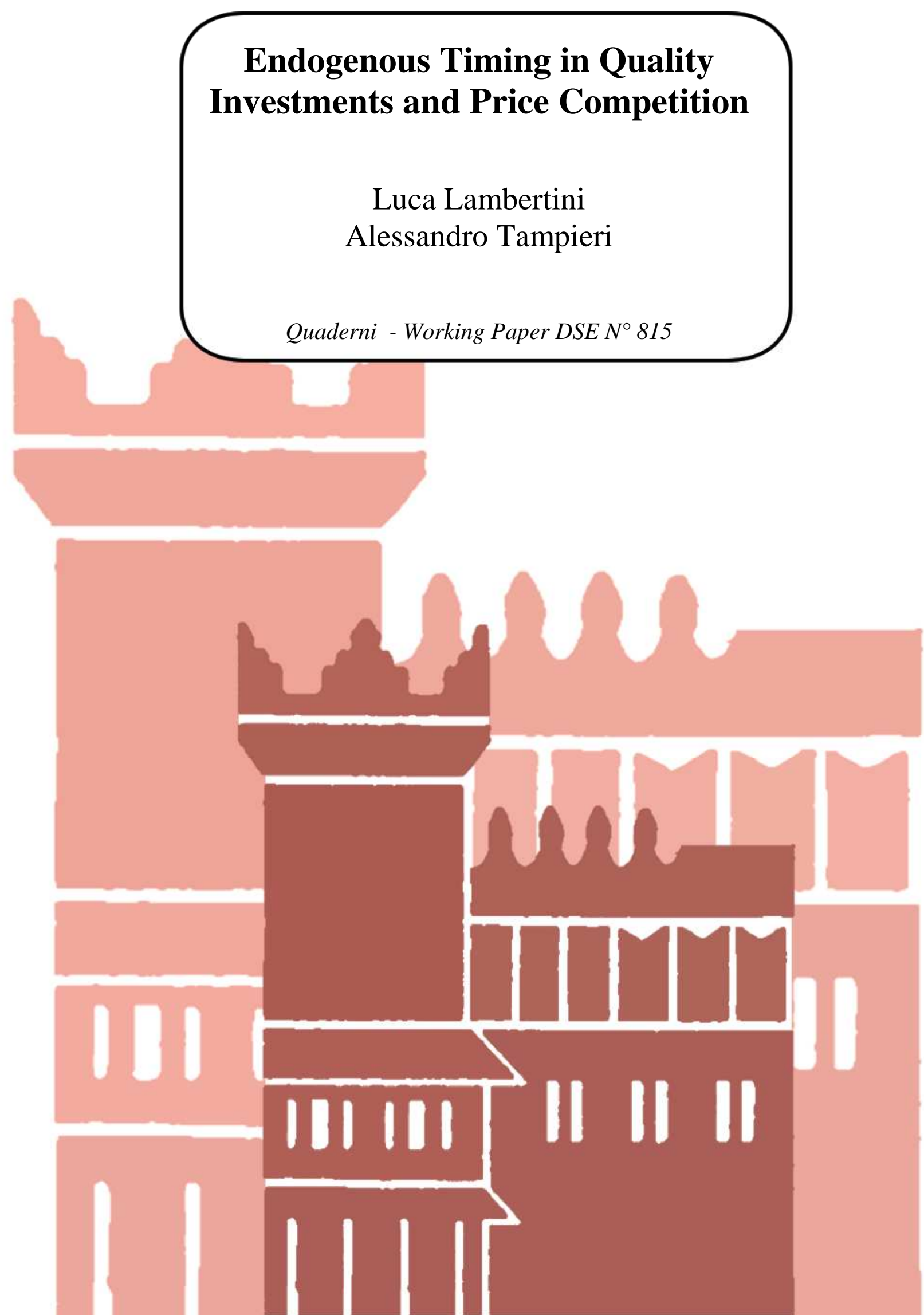




\title{
Endogenous Timing in Quality Investments and Price Competition
}

\author{
Luca Lambertini ${ }^{\S}$ and Alessandro Tampieri ${ }^{\S}$ \\ $\S$ Department of Economics, University of Bologna \\ Strada Maggiore 45, 40125 Bologna, Italy \\ luca.lambertini@unibo.it; alessandro.tampieri@unibo.it
}

March 5, 2012

\begin{abstract}
We modify the price-setting version of the vertically differentiated duopoly model by Aoki (2003) by introducing an extended game in which firms noncooperatively choose the timing of moves at the quality stage. Our results show that there are multiple equilibria in pure strategies, in which firms always select sequential play at the quality stage. We also investigate the mixed-strategy equilibrium, revealing that the probability of generating outcomes out of equilibrium is higher than its complement to one. In the alternative of full market coverage, we show that the quality stage is solved in dominant strategies and therefore the choice of roles becomes irrelevant as the Nash and Stackelberg solutions coincide.
\end{abstract}

JEL codes: C73, L13

Keywords: endogenous timing, product quality, market coverage. 


\section{Introduction}

In this note, we study the equilibria of a vertically differentiated Bertrand duopoly where the timing of moves at the quality stage is endogenised via an extended game with observable delay à la Hamilton and Slutsky (1990). In particular, we take this standpoint to complement the analysis carried out by Aoki (2003), in which

- variable production costs are nil and quality improvements hinge upon fixed costs,

- the quality stage is assumed to be either simultaneous or sequential and the timing cannot be decided by firms and

- in the sequential case, the leader and follower's roles are predetermined while the choice between high and low quality is endogenous. This yields that the leader will supply a higher quality than the rival's (see Aoki, 2003, Proposition 2, p. 659).

Here instead we assume as exogenous the location of firms along the quality spectrum, while endogenising the distribution of roles to be taken at the quality stage through a pre-play stage preceding the quality investment phase. In this respect, our analysis is based on d'Aspremont and GérardVaret (1980) and Hamilton and Slutsky (1990), according to whom a game is Stackelberg-solvable if there exists a Stackelberg equilibrium that Paretodominates the Nash solution. ${ }^{1}$

Our analysis consider two alternative cases separately. In the first (already considered by Aoki, 2003), partial market coverage prevails. If so,

\footnotetext{
${ }^{1}$ The analysis of the same vertically differentied industry under Cournot competition is in Lambertini and Tampieri (2012), where it is shown that there exists a unique subgame perfect equilibrium where sequential play obtains in the quality stage and the low-quality firm takes the leader's role.
} 
then from the analysis of the quality stage, there emerges that both firms' best replies are increasing. This produces two pure-strategy equilibria, both charcterised by sequential play, posing a coordination problem. Additionally, there also exists a Nash equilibrium in mixed strategies, whose characterisation reveals that the probability of generating an out-of-equilibrium outcome (that is, committing the mistake of playing simultaneously) is indeed strigtly higher than the probability of playing one of the two Nash equilibria in pure strategies. In the second scenario, we investigate the same issue under full market coverage (not considered by Aoki, 2003). This reveals that the quality stage has a unique subgame perfect equilibrium at the intersection of dominant strategies where the Nash and Stackelberg solutions coincide, since best reply functions are orthogonal in that stage. This implies that, in the presence of full market coverage, the choice of timing becomes indeed immaterial.

\section{The model}

We consider a duopoly market for vertically differentiated products supplied by single-product firms. The demand side is modelled à la Mussa and Rosen (1978). There is a continuum of consumers whose types are identified by $\theta$, uniformly distributed with density equal to one in the interval $\left[\theta_{0}, \theta_{1}\right]$, with $\theta_{0}=\theta_{1}-1$, where $\theta$ represents the consumers' marginal willingness to pay for quality. Each consumer is assumed to buy at most one unit of the vertically differentiated good in order to maximise the following surplus function:

$$
U=\theta q_{i}-p_{i},
$$

where $q_{i} \in[0, Q]$ indicates the quality of the product and $p_{i}$ is the market price at which that variety is supplied by firm $i=H, L$, with $q_{H} \geq q_{L}$. Therefore, the consumer who is indifferent between $q_{H}$ and $q_{L}$ is identified 
by the level of marginal willingness to pay $\widehat{\theta}$ that solves $\widehat{\theta} q_{H}-p_{H}=\widehat{\theta} q_{L}-p_{L}$, and therefore $\widehat{\theta}=\left(p_{H}-p_{L}\right) /\left(q_{H}-q_{L}\right)$. Hence, market demand for the high-quality good is $x_{H}=\theta_{1}-\widehat{\theta}$. For the moment, we assume partial market coverage, so that there exists a consumer indifferent between buying $q_{L}$ or not buying at all, identified by the marginal willingness to pay $\widetilde{\theta}$ solving $\widetilde{\theta} q_{L}-p_{L}=0$, whereby $\widetilde{\theta}=p_{L} / q_{L}$ and the demand for the inferior variety is $x_{L}=\widehat{\theta}-\widetilde{\theta}$. As in Aoki (2003), variable costs are assumed away and firms incur in convex fixed costs of quality improvement $C_{i}=k q_{i}^{n}, i=H, L$, where $k>0$ and $n \geq 2$. Hence profit functions are $\pi_{H}=p_{H} x_{H}-k q_{H}^{n}$ and $\pi_{L}=p_{L} x_{L}-k q_{L}^{n}$.

Aoki (2003) analyses a two-stage non cooperative game in which, taking the timing of moves at the quality stage as given, firms first (i) set qualities, and then (ii) set prices, the second stage taking place under simultaneous play. From this structure, Aoki (2003, Proposition 2, p. 659) draws the conclusion that, if information at the quality stage is perfect, then the leader is the high-quality firm.

To offer an alternative persepctive, we depart from Aoki's setup in proposing a game consisting of three stages, in which firms' identities along the quality ladder are given at the outset. In the first stage, firms choose the timing to be followed in the second stage, where qualities are set, and then in the third stage simultaneous Bertrand competition takes place. The solution concept is the subgame perfect equilibrium by backward induction.

The first stage is a pre-play stage à la Hamilton and Slutsky (1990), in which, under complete, symmetric and imperfect information, firms play a discrete strategy game represented in Matrix 1. 


\begin{tabular}{|c|c|c|}
\hline & \multicolumn{2}{|c|}{$L$} \\
\hline & $F$ & $S$ \\
\hline$H \quad F$ & $\pi_{H}^{N} ; \pi_{L}^{N}$ & $\pi_{H}^{S L} ; \pi_{L}^{S F}$ \\
\hline$S$ & $\pi_{H}^{S F} ; \pi_{L}^{S L}$ & $\pi_{H}^{N} ; \pi_{L}^{N}$ \\
\hline
\end{tabular}

Matrix 1

Actions $F$ and $S$ stand for "first" or "second", and refers to the choice of roles in the quality stage, while superscripts $N, S L$, and $S F$ stand for Nash, Stackelberg leader and Stackelberg follower, respectively. If firms select the same strategy - along the main diagonal - then the second-stage quality game is simultaneous. Conversely, along the secondary diagonal, the quality stage is going to be solved à la Stackelberg.

\section{Results}

To begin with, we recollect Aoki (2003)'s results to establish how they change by taking as exogenous the location of firms in the quality spectrum and endogenising the timing of quality investment. Aoki (2003) shows that, under Bertrand competition, the best replies at the quality stage are increasing and discontinuous if both firms are still to choose their relative positions along the quality spectrum (Aoki, 2003, Lemma 2, p. 657). By the same token, there are two pure-strategy (and identical) Nash equilibria to the simultaneous quality choice game (Aoki, 2003, Proposition 1, p. 658) and one Stackelberg equilibrium in the sequential game where the leader produces the higher quality (Aoki, 2003, Proposition 2, p. 659). The results are based on the fact that the high and low quality roles are endogenous, while the timing of moves is exogenously given.

Conversely, by assuming as exogenous whether a firm provides the high or low quality, the best response functions become continuous and firms may 
indeed have the possibility of non cooperatively choosing the respective roles in the quality stage via an extended game with observable delay à la Hamilton and Slutsky (1990).

The results can be qualitatively assessed by examining Figure 1, which shows the map of best replies and the isoprofit curves. The Nash equilibrium (point $N$ ) under simultaneous play yields to both firm $L$ and firm $H$ a lower profit as compared to either of the Stackelberg equilibria (points $S_{L}$ and $S_{H}$ ), so that the following chains of inequalities hold:

$$
\begin{aligned}
& \pi_{H}^{S F}>\pi_{H}^{S L}>\pi_{H}^{N} \\
& \pi_{L}^{S F}>\pi_{L}^{S L}>\pi_{L}^{N}
\end{aligned}
$$

Hence, the timing game depicted in Matrix 1 yields two pure-strategy equilibria along the secondary diagonal, according to which firm is leader. It is noteworthy to point out that, in each equilibrium the follower obtains the highest profit. This is perfectly in line with Hamilton and Slutsky (1990), according to which if a firm has a decreasing (resp., increasing) reaction function, it will prefer to move first (resp., second) (see Hamilton and Slutsky, 1990, Theorem V, p. 38). The foregoing discussion can be summarised in

Proposition 1 With Bertrand competition, the three stage game has two pure-strategy subgame perfect equilibria along the secondary diagonal, in which firms choose to play sequentially at the quality stage. 
Figure 1. The map of best replies with partial market coverage.

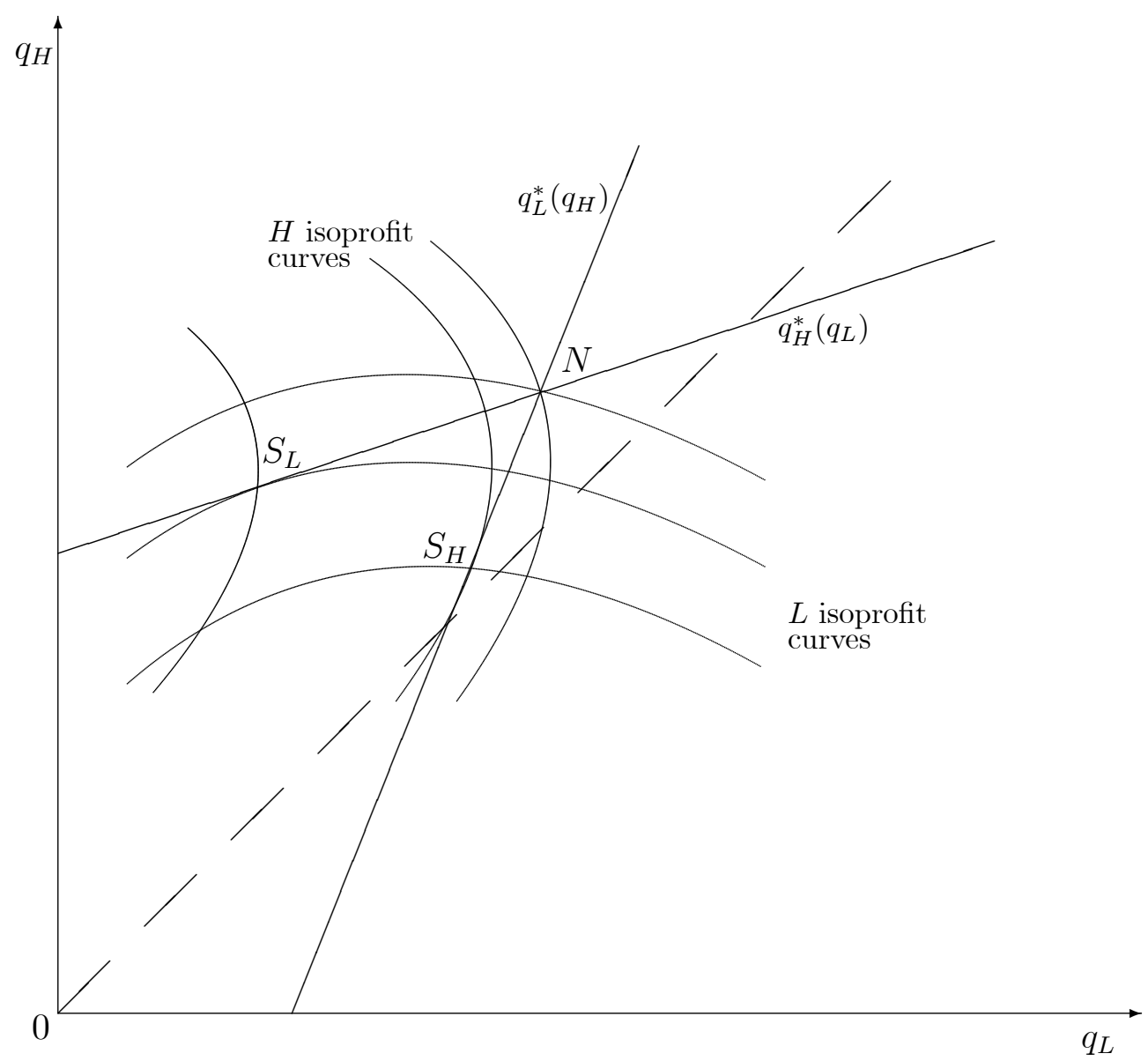

The multiplicity problem highlighted by the above Proposition also entails that there exists a mixed-strategy Nash equilibrium which is worth investigating so as to ascertain the relative probabilities of playing one of the two pure-strategy equilibria along the secondary diagonal of Matrix 1, or instead committing a mistake by generating either outcome along the main diagonal.

Given the a priori symmetry of the model, we can solve the mixed strategy Nash equilibrium of the first stage of the game from the standpoint of firm 
$i=H, L$, whose task is to attach probabilities $\mathfrak{p}_{i} \in[0,1]$ to strategy $F$ and $1-\mathfrak{p}_{i}$ to strategy $S$, respectively, so as to make firm $j \neq i$ indifferent between its own pure strategies. This implies solving the following equation:

$$
\mathfrak{p}_{i} \pi_{j}^{N}+\left(1-\mathfrak{p}_{i}\right) \pi_{j}^{S L}=\mathfrak{p}_{i} \pi_{j}^{S F}+\left(1-\mathfrak{p}_{i}\right) \pi_{j}^{N}
$$

w.r.t. $\mathfrak{p}_{i}$, delivering

$$
\mathfrak{p}_{i}^{*}=\frac{\pi_{j}^{S L}-\pi_{j}^{N}}{\pi_{j}^{S F}+\pi_{j}^{S L}-2 \pi_{j}^{N}} \in(0,1) .
$$

Using (4), we can quickly estabilish that the probability of playing either $(F, S)$ or $(F, S)$ is

$$
\mathfrak{P}((F, S) \cup(S, F))=\mathfrak{p}_{H}^{*}\left(1-\mathfrak{p}_{L}^{*}\right)+\left(1-\mathfrak{p}_{H}^{*}\right) \mathfrak{p}_{L}^{*}
$$

while the probability of playing along the main diagonal is

$$
\mathfrak{P}((F, F) \cup(S, S))=\mathfrak{p}_{H}^{*} \mathfrak{p}_{L}^{*}+\left(1-\mathfrak{p}_{H}^{*}\right)\left(1-\mathfrak{p}_{L}^{*}\right),
$$

with

$$
\begin{gathered}
\mathfrak{P}((F, F) \cup(S, S))-\mathfrak{P}((F, S) \cup(S, F))= \\
\frac{\left(\pi_{H}^{S F}-\pi_{H}^{S L}\right)\left(\pi_{L}^{S F}-\pi_{L}^{S L}\right)}{\left(\pi_{H}^{S F}+\pi_{H}^{S L}-2 \pi_{H}^{N}\right)\left(\pi_{L}^{S F}+\pi_{L}^{S L}-2 \pi_{L}^{N}\right)}>0 .
\end{gathered}
$$

This result holds irrespective of the degree of vertical differentiation to be chosen in the second stage, as long as $q_{H}>q_{L}$, and implies:

Corollary 2 The mixed-strategy Nash equilibrium at the timing stage reveals that the probability of committing a mistake is strictly higher than the probability of playing either pure-strategy Nash equilibrium, for all admissible pair of qualities $q_{H}>q_{L}$.

The problem of multiple equilibria disappears if full market coverage obtains. In what follows, we retain the set of assumptions introduced above, 
except that now the market demand for the low-quality good is $x_{L}=\widehat{\theta}-$ $\left(\theta_{1}-1\right)$. The profit functions at the third stage are thus defined as

$$
\pi_{H}=p_{H}\left(\theta_{1}-\frac{p_{H}-p_{L}}{q_{H}-q_{L}}\right)-k q_{H}^{n} ; \pi_{L}=p_{L}\left(\frac{p_{H}-p_{L}}{q_{H}-q_{L}}-\theta_{1}+1\right)-k q_{L}^{n} .
$$

From the first order conditions at the market stage, we obtain the following equilibrium prices:

$$
\begin{aligned}
& p_{H}=\frac{\left(\theta_{1}+1\right)\left(q_{H}-q_{L}\right)}{3}, \\
& p_{L}=\frac{\left(2-\theta_{1}\right)\left(q_{H}-q_{L}\right)}{3},
\end{aligned}
$$

implying the constraint $\theta_{1} \in(1,2)$. Full market coverage is admissible if and only if the individual of the lowest type $\theta_{0}$ obtains a non-negative surplus from the consumption of the low-quality good, i.e., if:

$$
\left(\theta_{1}-1\right) q_{L}-p_{L} \geq 0
$$

Plugging (10) into (11), the latter turns out to be satified by all

$$
q_{L} \leq \frac{\left(2 \theta_{1}-1\right) q_{L}}{2-\theta_{1}}
$$

with

$$
\frac{\left(2 \theta_{1}-1\right) q_{L}}{2-\theta_{1}}>q_{L}
$$

in the admissible range of $\theta_{1}$. This yields the following Lemma:

Lemma 3 Given $\theta_{1} \in(1,2)$, full market coverage is admissible for all

$$
q_{H} \in\left(q_{L}, \frac{\left(2 \theta_{1}-1\right) q_{L}}{2-\theta_{1}}\right) .
$$

We turn now on the quality stage. The relevant profit functions are:

$$
\begin{aligned}
\pi_{H} & =\frac{\left(\theta_{1}+1\right)^{2}\left(q_{H}-q_{L}\right)}{9}-k q_{H}^{n}, \\
\pi_{L} & =\frac{\left(2-\theta_{1}\right)^{2}\left(q_{H}-q_{L}\right)}{9}-k q_{L}^{n} .
\end{aligned}
$$


The first order conditions for non cooperative profit maximisation are:

$$
\begin{gathered}
\frac{\partial \pi_{H}}{\partial q_{H}}=\frac{\left(\theta_{1}+1\right)^{2}}{9}-9 n k q_{H}^{n-1}=0 \\
\frac{\partial \pi_{L}}{\partial q_{L}}=\frac{\left(2-\theta_{1}\right)^{2}}{9}-9 n k q_{L}^{n-1}=0 .
\end{gathered}
$$

Given that (16-17) do not allow for a fully analytical characterisation of Nash and Stackelberg equilibria, we investigate the solution of the quality stage through the map of the reaction functions, implicitly determined by the above FOCs. In particular, Bulow et al. (1985) show that the nature of strategic interaction is completely determined by the sign of the partial derivatives of FOCs with respect to the competitor's quality, which indicate the slopes of reaction functions $q_{i}^{*}\left(q_{j}\right), i, j=H, L, i \neq j$. It is straightforward to check that:

$$
\begin{aligned}
& \frac{\partial q_{H}^{*}\left(q_{L}\right)}{\partial q_{L}}=\frac{\partial^{2} \pi_{H}}{\partial q_{H} \partial q_{L}}=0 \\
& \frac{\partial q_{L}^{*}\left(q_{H}\right)}{\partial q_{H}}=\frac{\partial^{2} \pi_{L}}{\partial q_{L} \partial q_{H}}=0
\end{aligned}
$$

so that each firm has a dominant strategy to be adopted irrespective of the competitor's quality choice. This also implies that the Nash and Stackelberg equilibrium in the second stage coincide. Hence the timing plays no role in determining the investment in quality both for the $H$ and $L$ firm, and the first stage disappears altogether (equivalently, one can say that, under full coverage, the three-stage game collapses into a two stage game as the choice of roles becomes immaterial).

Figure 2 shows the map of the best replies and the respective isoprofit curves. Clearly, the point where the reaction functions intersect (i.e., the Nash solution) corresponds to the point where the isoprofit curve of each firm (playing as the Stackelberg leader) is tangent to the reaction function of the respective competitor (in the follower's role). This leads to the following Proposition: 
Proposition 4 With full market coverage, the game has a unique subgame perfect equilibrium at the intersection of dominant strategies, where the Nash and Stackelberg solutions coincide.

Figure 2. The map of best replies with full market coverage.

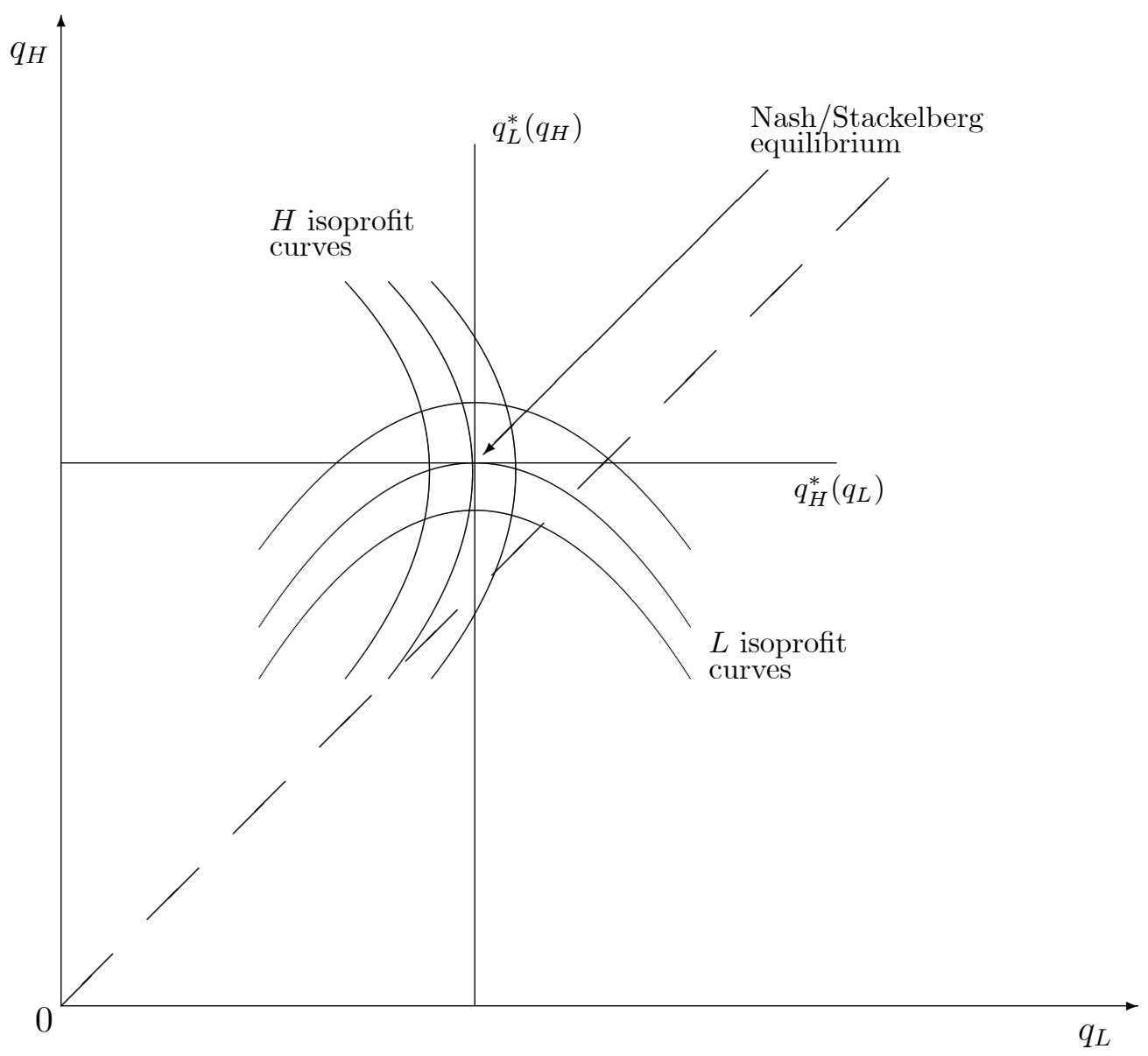

\section{Concluding remarks}

We have revisited Aoki's (2003) analysis of Stackelberg vs Nash equilibria in a game describing the choice of roles by firms supplying vertically differenti- 
ated goods and competing in prices. Analysing this issue in the framework of an extended game with observable delay (Hamilton and Slutsky, 1990) we have shown that, if partial market coverage prevails, then (i) multiple equilibria obtain - all of them involving sequential play - in pure strategies, so that (ii) the analysis of mixed strategy is informative, as there emerges that committing mistakes is more likely than playing either pure-strategy equilibrium. Alternatively, under full coverage, the multiplicity issue completely vanishes, replaced by unicity of equilibrium at the intersection of dominant strategies. 


\section{References}

[1] Aoki, R. (2003). Effect of Credible Quality Investment with Bertrand and Cournot Competition. Economic Theory 21: 653-672.

[2] Bulow, J., Geanakoplos, J. and Klemperer, P. (1985). Multimarket Oligopoly: Strategic Substitutes and Complements. Journal of Political Economy 93: 488-511.

[3] d'Aspremont, C. and Gérard-Varet, L.-A. (1980). Stackelberg-Solvable Games and Pre-Play Communication. Journal of Economic Theory 23: 201-217.

[4] Hamilton, J. and Slutsky, S. (1990). Endogenous Timing in Duopoly Games: Stackelberg or Cournot Equilibria. Games and Economic Behavior 2: 29-46.

[5] Lambertini, L. and Tampieri, A. 2012. Low-quality leadership in a vertically differentiated duopolywith Cournot competition. Economics Letters 115: 396-398.

[6] Mussa, M. and Rosen, S. (1978). Monopoly and Product Quality. Journal of Economic Theory 18: 301-317. 


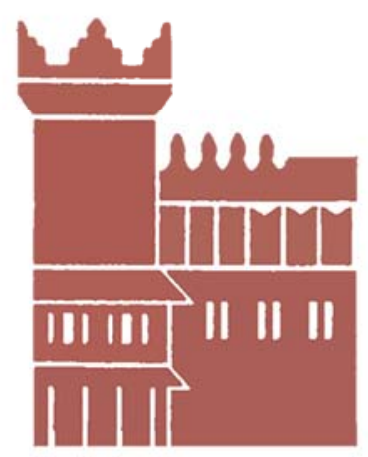

Alma Mater Studiorum - Università di Bologna DEPARTMENT OF ECONOMICS

Strada Maggiore 45

40125 Bologna - Italy

Tel. +39051 2092604

Fax +390512092664

http://www.dse.unibo.it 